\title{
Lineamientos estratégicos para la formación en liderazgo militar desde una perspectiva de género ${ }^{1}$
}

https://doi.org/10.21830/9789585241459.09

\author{
Lyria Esperanza Perilla Toro ${ }^{2}$ \\ Escuela Militar de Cadetes "General José María Córdova” \\ Leidy Johana Cabrera Cabrera ${ }^{3}$ \\ Escuela Militar de Cadetes "General José María Córdova”
}

\section{Resumen}

Antecedentes: la incorporación de mujeres de arma al Ejército Nacional en la última década creó la necesidad de comprender el liderazgo militar como eje de formación fundamental para la institución castrense. Objetivo: presentar una propuesta de lineamientos para la formación en liderazgo militar que contempla los actuales desarrollos sobre la perspectiva de género y el liderazgo femenino. Materiales y métodos: se recolectó información sobre las creencias y actitudes acerca del ejercicio y la formación en liderazgo militar a través de un cuestionario, entrevistas y grupos focales. También se realizó una revisión documental de tipo institucional y teórico sobre asuntos curriculares. Resultados: se proponen competencias de conocimientos, metodológicas, participación y personales, con respecto a la relación entre liderazgo y género, en el nivel macrocurricular, así como pautas para los procesos de enseńanza-aprendizaje, en el nivel microcurricular. Conclusiones: se espera que, en las condiciones actuales, en las cuales existen

1 Este capítulo contiene parte de los resultados del proyecto de investigación Lineamientos Estratégicos para el Desarrollo de Procesos de Formación en Liderazgo Militar desde una Perspectiva de Género en la Escuela Militar de Cadetes «General José María Córdova» y del proyecto Enfoques diferenciales en el Ejército Nacional: una Propuesta desde las Ciencias Militares, del Grupo de Investigación en Ciencias Militares de la Escuela Militar de Cadetes "General José María Córdova” (ESMIC), registrado con el código COL0082556 de Minciencias. El primer proyecto contó con financiación por parte de la ESMIC y de Minciencias, a través de la convocatoria No. 784 "Programa de estancias postdoctorales beneficiarios Colciencias 2017". Los puntos de vista y los resultados de este capítulo pertenecen a las autoras y no reflejan necesariamente los de las instituciones participantes.

2 Doctora en psicología de la Universidad de los Andes y magíster en Administración y Psicóloga de la Universidad Nacional de Colombia. Investigadora posdoctoral del Grupo de Investigación en Ciencias Militares de la Escuela Militar de Cadetes “General José María Córdova”. Orcid: https://orcid.org/0000-0002-0856-456X. Contacto: le.perilla21@uniandes.edu.co

3 Magíster y especialización en Docencia e Investigación Universitaria de la Universidad Sergio Arboleda. Trabajadora Social de la Universidad de La Salle e investigadora del Grupo de Investigación en Ciencias Militares de la Escuela Militar de Cadetes “General José María Córdova”. Orcid: http://orcid.org/00000002-6398-6933. Contacto: leidy.cabrera@esmic.edu.co 
diferencias de género en la carrera militar como profesión, estos lineamientos contribuyan a generar un modelo de liderazgo militar exitoso para la formación integral de los futuros oficiales.

Palabras clave: formación; liderazgo femenino; liderazgo militar; lineamientos curriculares; perspectiva de género.

\section{Introducción}

Con la incorporación de mujeres de arma al Ejército Nacional en la última década se han generado nuevos escenarios que plantean la necesidad de aproximarse al liderazgo militar — eje de formación fundamental para la institución castrensedesde los actuales desarrollos sobre la perspectiva de género y el liderazgo femenino. De acuerdo con el Comando General de las Fuerzas Militares (2015), el género se refiere a las características de comportamiento y los roles socialmente atribuidos a hombres y mujeres en determinados contextos históricos, más allá de sus diferencias biológicas, y que contribuyen a definir las responsabilidades, oportunidades y barreras de unos y otras. El género no se refiere solo a hombres y mujeres en particular, sino también a las relaciones entre ellos. En este sentido, para Formujer (2003), la perspectiva de género alude a

un instrumento para acercarse a mirar la realidad, poniendo en cuestión las relaciones de poder que se establecen entre los géneros y en las relaciones sociales en general. [...]. [También permite] desnaturalizar la percepción que se tiene del ser varón o mujer y atribuir a sus roles y atributos un carácter socialmente construido. (p. 26)

Por lo tanto, esta perspectiva puede aplicarse en cualquier ámbito de la actividad humana como lo es, en este caso, la educación.

El liderazgo femenino, tal como lo definió Kaufmann (1996), es aquel que destaca la parte emocional y humana en la dirección de las organizaciones, de tal forma que crea un modo de trabajar principalmente reticular, a diferencia del modelo masculino, en el que prima la racionalidad científica y el orden social jerárquico. En concordancia con las características señaladas sobre la perspectiva de género, no se debe confundir con el liderazgo ejercido por las mujeres, pues como, lo aclara Medina-Vicent (2015), no se trata de que estas lideren diferente por el hecho de ser mujeres, sino que los distintos procesos de socialización han conducido a una identidad de género concreta para ellas, que implica constituirse en seres sensibles, emotivos y cooperativos. Tales cualidades se van a ver reflejadas en una particular forma de liderar, diferente a la del promedio de los hombres, 
cuya socialización está asociada con características como el control, la agresividad, la independencia, etc., las cuales corresponden al modelo masculino de liderazgo.

Específicamente, este estudio caracterizó desde una perspectiva de género el ejercicio y la formación en liderazgo militar para cadetes y subtenientes de la Escuela Militar de Cadetes “General José María Córdova” (ESMIC), a partir de información recolectada con un cuestionario, grupos focales y entrevistas. Asimismo se revisó la literatura sobre temas de género, currículo y liderazgo, los manuales de doctrina y lineamientos educativos de las Fuerzas Armadas, así como los documentos curriculares del programa de Ciencias Militares de la ESMIC. Este análisis sirvió como insumo para definir los lineamientos estratégicos de formación que se presentan en este documento.

En este contexto, el currículo es un eje articulador que puede integrar el liderazgo y su perspectiva de género como elementos fundamentales de los procesos de formación de los oficiales del Ejército Nacional. Dado que por su complejidad existen diversas formas de abordar el currículo, en este trabajo se analiza desde una perspectiva renovadora de las ciencias sociales y se comprende como una construcción cultural y social, dinámica y flexible que orienta el proceso educativo en todas sus dimensiones y define pautas para el ejercicio pedagógico (Avendaño \& Parada, 2013). Desde su posibilidad transformadora se plantea como un eje articulador que "convoca al diálogo de diferentes actores alrededor de las realidades educativas, con el fin de debatir, acordar y contrastar diversas perspectivas y experiencias" (Gimeno, 2010, p. 11) que permitan generar procesos de educación integrales, lo cual se ve reflejado en los lineamientos que cada institución educativa propone. Los lineamientos curriculares son entonces la directriz o pauta de acción orientada a definir elementos claves para la renovación curricular. En el caso específico de este trabajo, el liderazgo con perspectiva de género, la renovación se refiere a motivar la estructuración de escenarios académicos en permanente análisis a través de un proceso de construcción y transformación multifacético.

\section{Marco teórico}

\section{Liderazgo femenino}

El estilo de liderazgo se entiende como la forma en que los líderes estructuran su conducta cuando se desempeñan como tales y, sobre todo, la forma en que es percibida por los colaboradores. Es así que se han planteado diversos estilos (autocrático, democrático, orientado a la tarea, a las relaciones, etc.) que forman parte 
del transaccional, el cual se diferencia del estilo transformacional planteado por Bass (1985), que caracteriza a aquellos líderes que producen efectos extraordinarios en sus seguidores, principalmente a través de su influencia personal.

Desde la aparición de este último estilo y de la presencia de mujeres en el ámbito organizacional, comenzó a haber un interés por las diferencias entre estas y los hombres en su forma de liderar, de tal manera que surgieron múltiples estudios de diferentes características y, por lo tanto, diversas conclusiones. De acuerdo con la síntesis de literatura que hace Cuadrado (2003), se evidencia una tendencia a percibir que los hombres emplean estilos más autocráticos y orientados a la tarea, en cuanto se relacionan con la dimensión instrumental de los estereotipos de género, es decir, la agresividad, la independencia, la racionalidad, etc., mientras que la tendencia en las mujeres sería hacia un liderazgo más democrático y orientado a las relaciones, de acuerdo con la dimensión comunal de los estereotipos, caracterizada por la comprensión, la sensibilidad, la generosidad, etc.

Sin embargo, los estereotipos de género constituyen la principal causa de las dificultades que han tenido las mujeres que ocupan cargos de liderazgo, en la medida en que su comportamiento contradice lo que es deseable de acuerdo con su género: sensibilidad, complacencia, comprensión, entre otros (García, 2014) y porque el modelo de liderazgo exitoso tradicionalmente ha correspondido con el estereotipo masculino (Boldry, Wood \& Kashy, 2001; Lupano, Castro \& Casullo, 2008).

Una de las raíces de los estereotipos de género se encuentra en los procesos de socialización que generan en las mujeres una forma de ver el mundo como una "red de conexiones", mientras que los hombres lo perciben como un "orden social jerárquico" (Cuadrado, 2003). Para autores como Medina-Vicent (2015), esta diferencia daría como resultado dos modelos de liderazgo diferentes: por un lado estaría el femenino, en el cual "se da un predominio de la participación en igualdad de condiciones que fortalece las relaciones entre los miembros del equipo, creando modos de trabajo reticulares" (p. 188), y por otro estaría el modelo de liderazgo masculino, en el cual primarían el éxito personal y la racionalidad científica en el logro de los objetivos estratégicos" (p. 188).

Esta autora señala, sin embargo, que existen otros factores que determinan el desarrollo del liderazgo femenino, como el sexo del líder, el cargo de quienes evalúan a los líderes, el predominio numérico de un sexo dentro de la organización, así como su actividad y el sector al cual pertenece, la cultura y las políticas organizacionales, entre otros. En este sentido, si bien el modelo tradicional ha gozado de mayor reco- 
nocimiento, el contexto organizacional se ha transformado en las últimas décadas y los valores masculinos ya no poseen la misma eficacia para su liderazgo, de tal forma que se piden características propias del modelo transformacional.

$\mathrm{Al}$ respecto cabe mencionar que diversos estudios reportan mayores puntajes para las mujeres en las dimensiones del liderazgo transformacional, el cual ha demostrado de manera consistente predecir mejor el desempeño individual, grupal y organizacional, y cuyas particularidades se relacionan con rasgos asociados al liderazgo femenino, como la cooperación, la sensibilidad y el poder compartido (Ramos, 2005). Finalmente, para efectos del presente documento, se debe destacar que las características del estilo de liderazgo transformacional también se han asociado con las que son requeridas para el éxito del liderazgo militar (Parada, 2015).

\section{Currículo en la educación superior}

Las instituciones de educación superior tienen entre sus principales retos responder a fenómenos sociales de la actualidad cada vez más demandantes, lo cual motiva una permanente preocupación por los procesos de enseñanza y su renovación, a la luz de las necesidades propias de cada una de las áreas de conocimiento. Desde esta perspectiva, este estudio asume el currículo como una categoría de análisis, dado que integra los horizontes de sentido y los principales retos para la formación de profesionales en ciencias militares, y por lo tanto es un eje fundamental para comprender sus dinámicas en el contexto de la ESMIC.

Los currículos, en palabras de Gimeno (1999), "aunque se establezcan como pautas obligatorias más o menos uniformes para todo el sistema escolar, siempre son objeto de interpretaciones moduladoras que concretan su contenido y sus objetivos, plasmándose en prácticas, de hecho, diversificadas" (p. 71), razón por la cual se convierte en eje articulador para los procesos de renovación, sobre todo en educación superior. Específicamente, se considera que la investigación del currículo es el medio a través del cual se organizan, seleccionan y distribuyen los contenidos culturales en la escuela, sin perder el horizonte de la eficiencia y la productividad, pero buscando consolidar en las personas, ideales más duraderos que la preparación para efectuar una técnica (Rojas \& Palacio, 2004).

En Colombia, el estudio del currículo forma parte de los procesos institucionales como componente estratégico para definir la educación superior. Así se evidencia en la Ley General de Educación (1994), en la cual se concibe como

el conjunto de criterios, planes de estudio, programas, metodologías, y procesos que contribuyen a la formación integral y a la construcción de la identidad cultural 
nacional, regional y local, incluyendo también los recursos humanos, académicos y físicos para poner en práctica las políticas y llevar a cabo el proyecto educativo institucional. (República de Colombia, 1994, Art. 76)

Como se observa, el currículo no solo define los elementos estratégicos que componen la formación, sino que debido a su nivel de importancia, también es uno de los elementos clave de evaluación para los procesos de acreditación institucional en educación superior.

El abordaje integral del currículo como eje articulador de los procesos de formación en educación superior ha avanzado desde una perspectiva tradicional y/o reduccionista —en la cual solamente se contempla la normatización—, hacia perspectivas renovadoras y críticas en las que se concibe la educación como un proceso social que debe responder a las exigencias de las nuevas realidades y centrarse en el ser humano como agente activo, comprometido y crítico. Esta perspectiva plantea entonces la necesidad de resignificar los procesos educativos para responder con las dinámicas actuales (Aktouf, 2001).

Con base en los diversos abordajes del currículo desde distintas áreas del conocimiento, las investigaciones sobre educación superior de la última década se han inclinado a realizar reflexiones, exponer casos y orientar discusiones en torno a distintas formas de concebir la gestión universitaria desde el currículo. Así, han definido varias categorías asociadas a la formación como derroteros en la planeación curricular, tales como: interdisciplinariedad, inclusión, competencias, flexibilización, innovación, entre otras (Roa, 2019).

\section{Lineamientos curriculares: el primer paso para el diseño curricular}

En el campo de la educación superior se reconoce que para hacer ajustes y transformaciones curriculares es determinante considerar el contexto, así como los intereses y los desafíos que se espera alcanzar desde la profesión y la disciplina estudiadas. Se trata de un proceso de diferentes etapas que involucra la investigación y que incluye: el diagnóstico, el diseño, la implementación y la evaluación, no como actividades unidireccionales, sino como un proceso de construcción que implica un ir y venir. Por esta razón es necesario contar con equipos de investigación permanentes que generen los espacios de discusión correspondientes y contribuyan a priorizar los ejes transversales de abordaje (Camacho et al., 2016; Malagón, Machado \& Rodríguez, 2013).

Para el Ministerio de Educación Nacional (MEN, 2016), los lineamientos curriculares son "las orientaciones epistemológicas, pedagógicas y curriculares que 
define el MEN con el apoyo de la comunidad académica educativa para apoyar el proceso de fundamentación y planeación de las áreas obligatorias y fundamentales definidas por la Ley General de Educación en su artículo 23”. En este sentido, la generación de lineamientos curriculares debe entonces analizar a fondo la dinámica de los procesos pedagógicos, para lo cual se debe revisar y evaluar permanente según los alcances disciplinares y profesionales que se emprendan y el contexto que enmarca las necesidades de la formación. En consecuencia, el diseño curricular va más allá de definir una estructura documental, sino que implica también llevar un seguimiento continuo y sistemático. Asimismo, cuando se hacen ajustes curriculares es fundamental vigilar que el diseño se ejecute realmente, mientras que su evaluación debe asumirse como un proceso con adecuadas estrategias e instrumentos para recoger observaciones e informaciones, y analizarlas con la participación de los diversos actores implicados (Roa, 2019).

Según Tovar y Sarmiento (2011), "uno de los elementos críticos en el diseño curricular es la responsabilidad compartida, lo que exige la participación de los diferentes actores, por lo cual se insiste en sugerir que se implementen estrategias que permitan la participación de estudiantes, egresados, empleadores, padres de familia, asociaciones gremiales, profesionales y grupos sociales" (p. 516). Las estrategias de participación de la comunidad académica en los diseños curriculares permiten avanzar desde una perspectiva instrumental hacia una perspectiva renovadora de la educación, en la que conceptos como liderazgo militar y perspectiva de género pueden tener espacio de revisión y resignificación según las propias dinámicas de los centros de formación militar.

\section{Integración curricular desde la perspectiva de género}

De acuerdo con Miranda (2012), la perspectiva de género "apunta hacia la distinción entre la diferencia sexual y los roles sociales que se construyen a partir de dicha diferencia. Su principal objetivo es la consecución de la igualdad de derechos y oportunidades entre varón y mujer, sin homogeneizarlos" (pp. 346-347). Al respecto, López (2007) señala que "la aplicación de la perspectiva de género tiene una dimensión política inevitable y no conviene reducirla a una mera técnica o herramienta: nos permite promover cambios sociales o, al menos, evitar seguir profundizando las discriminaciones de género" (p. 34). Por lo tanto, incorporar la perspectiva de género en la formación profesional en particular implica cuestionar situaciones de desigualdad que derivan de los estereotipos y roles de género, es decir, de factores sociales, culturales y educativos que son modificables. También 
implica la deconstrucción de masculinidades tradicionales que den paso a masculinidades alternativas en las que los hombres
se cuestionan sus roles y privilegios en las diferentes esferas de su desarrollo personal y que, en esa misma lógica, trabajen en contra de las violencias basadas en el género y todo tipo de discriminación de todas aquellas personas que no se enmarcan en la estructura binaria de los géneros. (Ministerio de Defensa Nacional, 2018, p. 18)

Por lo anterior, para introducir dicha perspectiva en los lineamientos curriculares se acogió el marco teórico utilizado para clasificar y definir las Competencias de Igualdad de Género (Mimbrero, Pallarès \& Cantera, 2017), desde el cual se retoman los tres niveles en los que se manifiestan los géneros. Según el enfoque Doing Gender,

en el Nivel sociocultural o socioestructural, el género funciona como agente, como un sistema de organización social que gobierna el acceso al poder y a los recursos. El Nivel interaccional o relacional, se visibiliza a través de las diferencias desde las que hombres y mujeres actúan y son tratados en las interacciones cotidianas. El Nivel individual o personal se muestra a través de la autorrepresentación femenina o masculina. (Mimbrero et al., 2017, p. 267)

También se retoma el concepto integrado de competencias que incluye tanto los conocimientos que forman parte del desempeńo y los atributos personales que contribuyen al logro, como los valores y demás características del contexto en el que se realiza el trabajo. Este concepto resulta muy adecuado frente a los planteamientos del Sistema Educativo de las Fuerzas Armadas (Sefa) acerca del currículo, que lo concibe como una construcción cultural y un modo de organizar una serie de prácticas educativas. $\mathrm{Al}$ respecto, señala que

la calidad de la educación y la enseñanza tienen que ver con el tipo de cultura que en ella se desarrolla, que obviamente cobra significado educativo a través de las prácticas y códigos que se traducen en procesos de aprendizaje para los estudiantes. Las prácticas educativas, tienen mucho que ver con los usos, tradiciones, técnicas y valores dominantes en el entorno de la realidad educativa (Ministerio de Defensa Nacional, 2010, p. 8-9).

Entender el currículo como una construcción cultural y reconocer la relevancia que tienen los valores del contexto educativo permite comprender aún más la trascendencia de introducir la perspectiva de género en la formación de competencias de liderazgo en un ámbito cuyas dinámicas se han estado transformando en los tres niveles señalados, como se verá más adelante. 


\section{Metodología}

El enfoque de la investigación fue de tipo mixto, que en palabras de Sampieri y Mendoza (2018) "representa un conjunto de procesos sistemáticos, empíricos y críticos de investigación que implican la recolección y análisis de datos cuantitativos y cualitativos, así como su integración y discusión conjunta” (p. 612). En este sentido, se utilizaron datos y técnicas de recolección de información de tipo cuantitativo y cualitativo.

El componente cuantitativo abordó los resultados de una caracterización sobre formación en liderazgo militar empleando información de la base de datos elaborada por Fernández-Osorio, Latorre y Mayorga (2018). En el componente cualitativo, que tiene especial énfasis en este capítulo, se hicieron, por un lado, cinco entrevistas a profundidad realizadas a cuatro subtenientes de las armas de Logística, Comunicaciones y Transporte, y a una teniente coronel de carrera administrativa de la Oficina de Género del Ejército Nacional. Por otro lado, se realizaron cinco grupos focales con cadetes de quinto nivel. Las transcripciones de las entrevistas y los grupos focales fueron procesadas a través del software de análisis de datos cualitativos Atlas. ti 7.

Asimismo, se aplicó la técnica de revisión documental, desde la cual se rastrearon documentos y literatura de tipo institucional, relacionada principalmente con fundamentos macro- y microcurriculares. Se asumieron como documentos de tipo macrocurricular aquellos que se enmarcan en los lineamientos generales de la institución y que se acogen a las indicaciones generales de la normatividad nacional vigente. Los documentos de tipo microcurricular se refieren al sistema conceptual como ordenador de la actividad en el aula y el elemento dinámico del currículo que constituye, para la institución educativa, momentos de verdad que ponen a prueba los discursos sobre la calidad, el perfil, la coherencia del programa y su currículo, al tiempo que establece y confronta la actividad en el aula con el encargo social institucional (Osorio, 2012). Los documentos revisados fueron: el Plan Estratégico del Sistema Educativo de las Fuerzas Armadas (Pese), 2007-2019; el Proyecto Educativo de las Fuerzas Armadas (Pefa), 2008; los Lineamientos Curriculares para las Fuerzas Militares del Sistema Educativo de las Fuerzas Armadas (Sefa), 2010; el Manual Fundamental del Ejército 6-22. Liderazgo, 2016 (Centro de Doctrina del Ejército [Cedoe], 2016); el Manual Fundamental de Referencia del Ejército 6-22. Liderazgo, 2017 (Cedoe, 2017); el Proyecto Educativo del Programa de Ciencias Militares, 2019, y los Contenidos Temáticos del Programa de Ciencias Militares, 2019. El tratamiento de la información obtenida en la revisión documental incluyó un análisis categorial y descriptivo a través de una matriz de análisis. 
Finalmente, y como producto del proceso de revisión documental y de las percepciones de las oficiales entrevistadas y los participantes de los grupos focales, se plantearon los lineamientos curriculares y se ubicaron en las categorías macrocurricular y microcurricular, como insumo para avanzar en el diseño curricular que integre el liderazgo militar desde una perspectiva de género.

\section{Resultados}

\section{Creencias y actitudes sobre la formación y el ejercicio militar en la ESMIC}

A continuación se presentan los resultados de las entrevistas en dos ejes fundamentales que definieron las experiencias de vida de las oficiales de arma que se encuentran actualmente en ejercicio de su profesión. En primer lugar, se destacan los motivadores intrínsecos y extrínsecos que fueron determinantes en la decisión por vincularse al Ejército Nacional y, en segundo lugar, los elementos que configuraron su formación militar y su ejercicio en campo.

\section{LA VINCULACIÓN}

La integración de la mujer en las Fuerzas Militares ha sido analizada desde diferentes perspectivas alineadas con el contexto social en el que se circunscriben. De acuerdo con Echavarría (1994), este proceso se relaciona con cuatro vectores que actúan de una forma conjunta:

El político, que propicia el reconocimiento de los derechos de la mujer y la igualdad entre todos los ciudadanos. El laboral, que va permitiendo y aceptando la igualdad con respecto al trabajo sin discriminación. El sociológico, que asume culturalmente una mayor aproximación entre los roles tradicionales que corresponden a cada sexo y una mayor equiparación de funciones y tareas. El militar, que va propiciando una mayor profesionalización del personal, y por lo tanto un modelo de carrera. (p. 67)

En este último se incluye la profesionalización de mujeres de arma como oficiales. Desde este vector militar, el Ejército Nacional ha propendido por consolidar su renovación, para lo cual ha generado nuevas apuestas que procuran comprender las dinámicas que trae la vinculación del personal militar femenino. Este proceso de comprensión inició, para el caso de este estudio, con preguntas sobre los principales motivadores que influyeron la decisión de vincularse a la institución.

De acuerdo con Ospina (2006), la motivación puede ser de dos tipos: por un lado, se destaca la motivación intrínseca, que "tiene su procedencia a partir del 
propio sujeto, está bajo su dominio y tiene como objetivo la experimentación de la autorrealización, por el logro de la meta, movido especialmente por la curiosidad y el descubrimiento de lo nuevo" (p. 159). Por otro lado, la motivación extrínseca se refiere al aprender no tanto por el gusto sino por las ventajas que ese conocimiento ofrece: "Es el efecto de acción o impulso que producen en las personas determinados hechos, objetos o eventos que las llevan a la realización de actividades, pero que proceden de fuera" (Ospina, 2006, p. 160).

Como se puede apreciar en la figura 1, al indagar por la motivación que tuvieron las oficiales para vincularse al Ejército Nacional se destacan tres categorías: en primer lugar está el impacto de los medios de comunicación (extrínseca) con publicidad relacionada (películas y series) que muestran los valores institucionales. La segunda categoría es la tradición familiar (intrínseca o extrínseca, según el caso), en la que destacan una historia de vida en la que se marca la importancia de continuar con una profesión que ha definido hitos importantes en las familias. Y, finalmente, la motivación desde experiencias de vida marcadas por la violencia y el conflicto armado interno (extrínseca), por lo cual centran su motivación en producir cambios desde la institución militar. Seguramente existen otros cambios importantes que generan las vinculaciones, pero en general se trata de experiencias personales que se ubican en áreas intrínsecas o extrínsecas según las experiencias de vida.

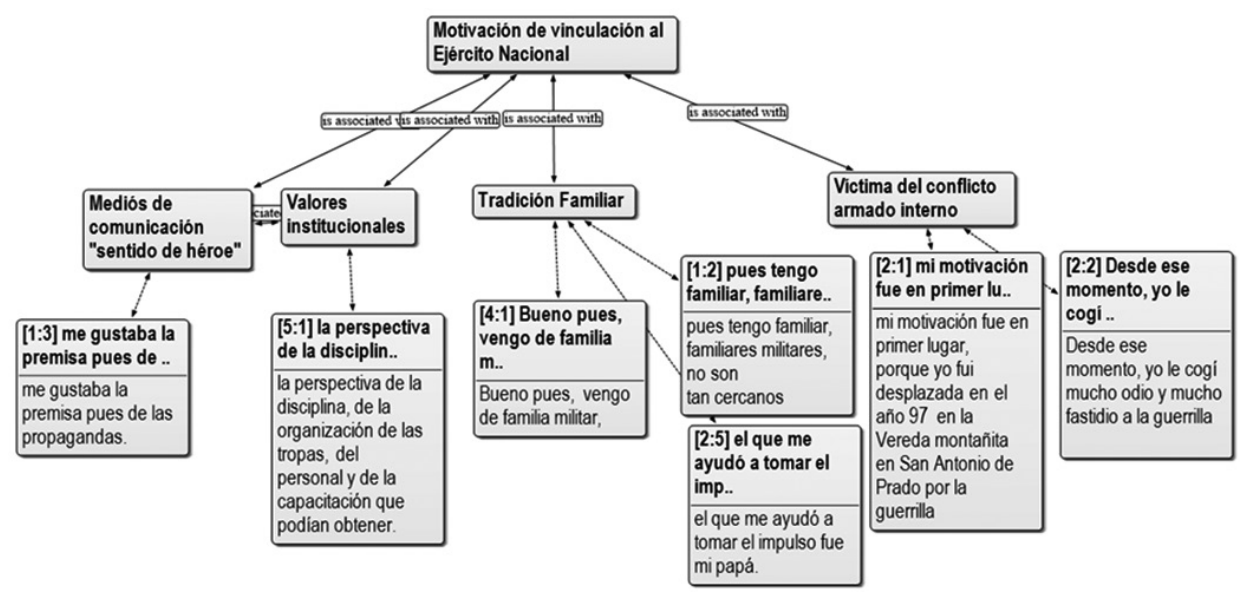

Figura 1. Motivación para la vinculación al Ejército Nacional. Fuente: Original de las autoras. 


\section{LA FORMACIÓN}

El proceso de educación que destacan las oficiales reconoce la formación práctica como eje principal de la enseńanza del liderazgo. Además señalan que la preparación académica no muestra una articulación y que por lo tanto no logra la recordación e impacto que debería tener. En este sentido, la formación práctica, vista desde la percepción de las oficiales, puede representarse en la figura 2.
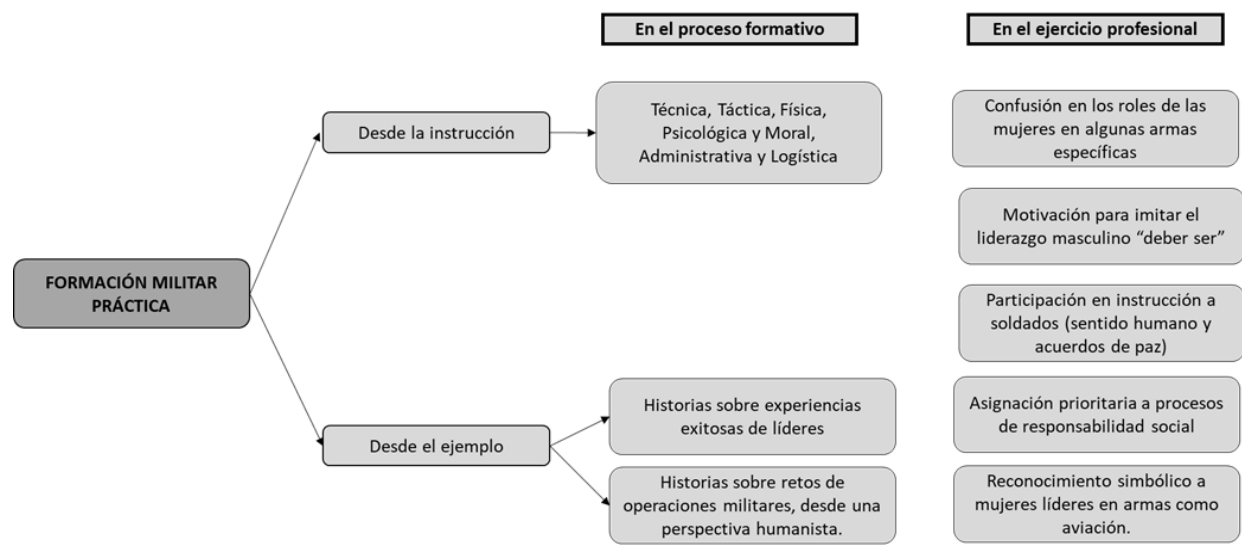

Figura 2. Estructura de interpretación de la formación militar práctica. Fuente: Original de las autoras.

La formación militar práctica está fundamentada en las áreas Técnica, Táctica, Física, Psicológica y Moral, Administrativa y Logística, con el fin de desarrollar las habilidades necesarias para cumplir su misión. Al respecto, lo que más resaltan las oficiales tiene que ver con la instrucción propiamente dicha que viven en sus procesos de entrenamiento militar. Se nombran experiencias y manejo de casos en los que destacan las habilidades técnico-militares.

Sin embargo, las oficiales perciben una confusión institucional frente a los roles que ellas deben ejercer en los campos de acción que tiene cada una de las armas. Esto ha generado resistencias sobre la participación de mujeres oficiales en campos específicos de arma como la Infantería y la Artillería. La mayor resistencia por parte de los hombres a que las mujeres pertenezcan a algunas armas o especialidades del Ejército puede responder, tal como lo expresa Camacho (2014), a las tensiones que generó la llegada de las cadetes a un entorno que, aunque las acogió, no estaba acostumbrado a su presencia y, por consiguiente, tiene que reorganizar su principal paradigma como creadora de oficiales del Ejército Nacional de Colombia, es decir, cuerpos guerreros que han sido configurados como máquinas para desem- 
peñarse eficazmente en las áreas de seguridad y defensa de la nación, y de acuerdo con necesidades particulares para tal fin.

El otro tipo de formación que también resaltan las oficiales tiene que ver con la formación desde el ejemplo, lo cual se encuentra en un área más personal y anecdótica con historias de operaciones que han desarrollado sus superiores. No se trata solo de los casos exitosos, sino que también resaltan la dimensión "humanista" de ese proceso, lo cual sitúa la experiencia de la persona como una fuente de conocimiento (Castillejo, 2015). Esa dinámica individual del relato es una experiencia personal e íntima que muchas veces se desliga del discurso histórico, y otras construye memoria colectiva que permite ser heredada; ambas terminan siendo concebidas como un pasado en el presente, una manera estratégica de concebirlo, lo que es fuente permanente de trabajo formativo en las aulas de clase.

Con respecto al ejercicio profesional existe confusión en los roles de las mujeres, pues muchas de ellas no realizan funciones propias del arma para la que fueron entrenadas, sino prioritariamente aquellas orientados a la responsabilidad social. En la formación y en acción profesional existe una constante motivación para imitar el liderazgo que ejercen los hombres, lo cual desdibuja el aporte que hacen ellas como mujeres. Como resultado existe un reconocimiento simbólico en algunas armas, pero según lo indicado, esto obedece a temas protocolarios y al cumplimiento de "la cuota femenina".

En conclusión, se percibe una igualdad en el ejercicio del liderazgo, sin que esto implique imitar la forma en que tradicionalmente se ha ejercido, lo cual abre un espacio para el liderazgo femenino en el ámbito militar. Motivar a través del ejemplo es considerado un rasgo característico del liderazgo militar que se evidencia más en las mujeres, quienes también presentan una mayor tendencia hacia la búsqueda del bienestar de los soldados. Las subtenientes también perciben contradicciones frente a su rol como líderes de unidades en áreas de combate y, en general, la percepción sobre las capacidades y responsabilidades militares es un factor que afecta la legitimación social del rol del liderazgo.

\section{Revisión documental}

El proceso de revisión documental incluyó el rastreo de información de fuentes primarias, manteniendo como eje transversal el lugar curricular que ocupa cada documento en la estructura de educación que se desarrolla, toda vez que el currículo se asume como el eje articulador del proceso de formación en liderazgo. De esta forma, la revisión documental, orientada desde el macrocurrículo y el micro- 
currículo, permitió reconocer aspectos estructurales del contenido en términos del lugar que ocupa el liderazgo, el liderazgo militar, y el género.

Como fuente primaria de base para el análisis de género se empleó el documento de Política Pública Sectorial de Transversalización del Enfoque de Género para el Personal Uniformado de la Fuerza Pública (2027-2018), específicamente en aspectos de formación. Este texto busca "promover la equidad de género en la Fuerza Pública, así como prevenir situaciones de violencias basadas en género en cada una de las Fuerzas Militares (FF. MM.) y la Policía Nacional, así como fortalecer el trabajo descentralizado alrededor de esta temática" (Ministerio de Defensa, 2018, p. 6). El liderazgo militar, como se observa en la tabla 1, configura un propósito central en la formación de oficiales del Ejército Nacional, lo cual se refleja en los documentos rectores que lo ubican en los lineamientos estructurales del proceso formativo. Asimismo, se encuentra que las políticas establecen el liderazgo militar como un requisito que se debe abordar desde niveles académicos y prácticos, y destacan los principios y atributos que lo componen. También especifican que es necesario desarrollar competencias exclusivas de liderazgo en las funciones de los oficiales militares, que se pueden materializar en acciones de entrenamiento como aplicaciones de maniobra y toma de decisiones en operaciones militares.

Tabla 1. Matriz de documentos macrocurriculares

\begin{tabular}{|c|c|c|c|}
\hline $\begin{array}{l}\text { Identificación del } \\
\text { documento }\end{array}$ & Descripción general & Liderazgo militar & Género \\
\hline $\begin{array}{l}\text { Plan Estratégico del } \\
\text { Sistema Educativo de las } \\
\text { Fuerzas Armadas (Pese), } \\
\text { 2007-2019 }\end{array}$ & $\begin{array}{l}\text { Presenta la formulación } \\
\text { y ejecución del Pefa }\end{array}$ & $\begin{array}{l}\text { Señala como una de } \\
\text { las políticas educativas } \\
\text { fortalecer la formación } \\
\text { para el liderazgo militar }\end{array}$ & No registra \\
\hline $\begin{array}{l}\text { Proyecto Educativo de } \\
\text { las Fuerzas Armadas } \\
\text { (Pefa) }\end{array}$ & $\begin{array}{l}\text { Define los principios } \\
\text { filosóficos, antropolóé } \\
\text { gicos, pedagógicos y } \\
\text { didácticos que inspiran } \\
\text { la visión y la misión del } \\
\text { Sefa. Es el referente para } \\
\text { que cada fuerza, basada } \\
\text { en los lineamientos y } \\
\text { políticas allí establecidas, } \\
\text { desarrolle el proyecto } \\
\text { educativo propio }\end{array}$ & $\begin{array}{l}\text { Señala como una de } \\
\text { las políticas educativas } \\
\text { fortalecer la formación } \\
\text { para el liderazgo militar }\end{array}$ & No registra \\
\hline
\end{tabular}




\begin{tabular}{|c|c|c|c|}
\hline $\begin{array}{l}\text { Identificación del } \\
\text { documento }\end{array}$ & Descripción general & Liderazgo militar & Género \\
\hline $\begin{array}{l}\text { Lineamientos } \\
\text { Curriculares de las } \\
\text { Fuerzas Militares - } \\
\text { Sistema Educativo de las } \\
\text { Fuerzas Armadas (Sefa), } \\
2010\end{array}$ & $\begin{array}{l}\text { Es el conjunto de } \\
\text { elementos interrela- } \\
\text { cionados para formar, } \\
\text { actualizar, capacitar, } \\
\text { instruir y entrenar a los } \\
\text { integrantes de las Fuerzas } \\
\text { Armadas }\end{array}$ & $\begin{array}{l}\text { Presenta como uno } \\
\text { de los requisitos de las } \\
\text { escuelas de formación } \\
\text { militar para el desarrollo } \\
\text { curricular que poten- } \\
\text { cien el desarrollo del } \\
\text { liderazgo en todos los } \\
\text { actores educativos. } \\
\text { Se constituye como una } \\
\text { de las competencias en } \\
\text { relación con el desarrollo } \\
\text { de la convivencia y la } \\
\text { interacción social. }\end{array}$ & No registra \\
\hline $\begin{array}{l}\text { Manual Fundamental } \\
\text { del Ejército 6-22. } \\
\text { Liderazgo, } 2016\end{array}$ & $\begin{array}{l}\text { Describe los requisitos } \\
\text { del modelo que se } \\
\text { deben seguir, enmar- } \\
\text { cados en atributos (el } \\
\text { ser) y competencias (el } \\
\text { hacer), de fácil aplica- } \\
\text { ción a través del Mando } \\
\text { Tipo Misión (MTM) } \\
\text { en el planeamiento, la } \\
\text { preparación, ejecución } \\
\text { y evaluación de las } \\
\text { Operaciones Terrestres } \\
\text { Unificadas (OTU). }\end{array}$ & $\begin{array}{l}\text { Atributos: carácter, } \\
\text { presencia e intelecto. } \\
\text { Competencias: liderar, } \\
\text { desarrollar y lograr. } \\
\text { Niveles: directo, organi- } \\
\text { zacional y estratégico }\end{array}$ & No registra \\
\hline $\begin{array}{l}\text { Manual Fundamental de } \\
\text { Referencia del Ejército } \\
\text { 6-22. Liderazgo, } 2017\end{array}$ & $\begin{array}{l}\text { Explica ampliamente } \\
\text { los principios de lide- } \\
\text { razgo estipulados en el } \\
\text { Manual Fundamental } \\
\text { del Ejército (MFE } \\
\text { 6-22). Liderazgo. En } \\
\text { este sentido, detalla los } \\
\text { niveles y condiciones } \\
\text { especiales del liderazgo y } \\
\text { describe los atributos y } \\
\text { competencias básicas del } \\
\text { líder en todos los niveles. }\end{array}$ & $\begin{array}{l}\text { Atributos: carácter, } \\
\text { presencia e intelecto. } \\
\text { Competencias: liderar, } \\
\text { desarrollar y lograr } \\
\text { Niveles: directo, organi- } \\
\text { zacional y estratégico }\end{array}$ & $\begin{array}{l}\text { Concepto: } \\
\text { la equidad } \\
\text { de género es } \\
\text { uno de los } \\
\text { aspectos de la } \\
\text { Constitución } \\
\text { que los líderes } \\
\text { militares en } \\
\text { todos sus } \\
\text { niveles deben } \\
\text { tener en } \\
\text { cuenta. }\end{array}$ \\
\hline
\end{tabular}




\begin{tabular}{|c|c|c|c|}
\hline $\begin{array}{l}\text { Identificación del } \\
\text { documento }\end{array}$ & Descripción general & Liderazgo militar & Género \\
\hline $\begin{array}{l}\text { Manual Fundamental } \\
\text { del Ejército 7-0. } \\
\text { Desarrollo de Líderes } \\
\text { y Entrenamiento de } \\
\text { Unidades, } 2016\end{array}$ & $\begin{array}{l}\text { Establece la función } \\
\text { del entrenamiento y la } \\
\text { capacitación del personal } \\
\text { militar y civil que ejerce } \\
\text { cargos de liderazgo en el } \\
\text { Ejército. }\end{array}$ & $\begin{array}{l}\text { Provee herramientas que } \\
\text { facilitan el desarrollo de } \\
\text { las OTU y orientan el } \\
\text { entrenamiento al desa- } \\
\text { rrollo de técnicas que } \\
\text { mejoren la aplicación de } \\
\text { la maniobra en el campo } \\
\text { de combate }\end{array}$ & No registra \\
\hline
\end{tabular}

Fuente: Original de las autoras.

La importancia de que las escuelas de formación desarrollen competencias en liderazgo militar está presente como eje transversal. En estudios sobre la percepción de los estudiantes-cadetes sobre este tema, se encuentra que "en su mayoría, consideran que aunque la formación académica brinda los elementos necesarios para ejercer el liderazgo militar, los elementos fundamentales son impartidos y fijados en la formación práctica” (Perilla \& Cabrera, 2018, p. 88), lo cual puede ser un punto de revisión curricular importante.

En cuanto a la perspectiva de género, el panorama es distinto, pues no se registra que se aborde en los documentos en general. Solo se alcanza una aproximación conceptual sobre equidad de género en el Manual de Fundamentación del Ejército para los líderes. Por esta razón, y de acuerdo con la Política Pública Sectorial de Transversalización del Enfoque de Género, es de vital importancia incluir en los lineamientos macrocurriculares la implementación de la perspectiva de género, la cual "deberá robustecer las acciones estratégicas de sensibilización, capacitación y formación del personal masculino de la Fuerza Pública, así como el desarrollo de estrategias de comunicación orientadas al cambio de imaginarios que permitan la construcción de una cultura libre de sexismos" (Ministerio de Defensa, 2018, p. 18), es decir, que no asigne "valores, capacidades y roles diferenciados a mujeres y hombres exclusivamente en función de su sexo" (Arraiz et al., 2015, p. 29).

Respecto a los contenidos ubicados en el nivel microcurricular (tabla 2), se encontró que el liderazgo militar se aborda en todas las materias o asignaturas que deben aprobar los oficiales. Su apuesta principal se evidencia especialmente en las competencias que se promueven en cuanto al liderazgo en sí mismo y el liderazgo ante la sociedad, que componen un análisis del sistema de valores individuales y sociales que enfrentan los oficiales en todos los espacios de ejercicio profesional. Un dato interesante para definir los lineamientos que se proponen en este trabajo es que 
documentos doctrinales como el MFE 6-22 plantean que los líderes deben equilibrar el éxito en el cumplimiento de la misión con la forma en que tratan y cuidan a los miembros de la organización. Por su parte, el género se aborda como una línea de investigación y de forma puntual en algunas asignaturas. Sin embargo, no se muestra la relación entre liderazgo y género como eje de análisis o abordaje curricular.

Tabla 2. Matriz de documentos microcurriculares

\begin{tabular}{|c|c|c|c|}
\hline $\begin{array}{l}\text { Identificación del } \\
\text { documento }\end{array}$ & Descripción general & Liderazgo militar & Género \\
\hline $\begin{array}{l}\text { Proyecto Educativo } \\
\text { del Programa de } \\
\text { Ciencias Militares, } \\
2019\end{array}$ & $\begin{array}{l}\text { Contextualiza un } \\
\text { marco institucional, } \\
\text { fija lineamientos } \\
\text { académicos y adminis- } \\
\text { trativos y determina } \\
\text { una proyección que } \\
\text { seńala el rumbo que } \\
\text { debe seguir el proceso } \\
\text { académico para lograr } \\
\text { los fines propuestos }\end{array}$ & $\begin{array}{l}\text { La Visión del } \\
\text { Programa enfatiza el } \\
\text { liderazgo basado en } \\
\text { la ética. Se plantea } \\
\text { la formación para } \\
\text { el liderazgo militar } \\
\text { del estudiante en } \\
\text { tres dimensiones: } \\
\text { ante sí mismo, ante } \\
\text { la institución y } \\
\text { ante la sociedad. La } \\
\text { primera se concreta } \\
\text { en un curso en el } \\
\text { segundo semestre } \\
\text { (Fundamentos de } \\
\text { Liderazgo), la segunda } \\
\text { en el Seminario Sabio } \\
\text { Caldas en cuarto } \\
\text { semestre, y la tercera } \\
\text { en séptimo semestre } \\
\text { con el curso de } \\
\text { Liderazgo Militar }\end{array}$ & $\begin{array}{l}\text { Se menciona la } \\
\text { equidad de género } \\
\text { como una de las } \\
\text { líneas de investiga- } \\
\text { ción del Grupo de } \\
\text { Investigación en } \\
\text { Ciencias Militares }\end{array}$ \\
\hline $\begin{array}{l}\text { Contenidos temáticos } \\
\text { del Programa de } \\
\text { Ciencias Militares, } \\
2019\end{array}$ & $\begin{array}{l}\text { Presenta los conte- } \\
\text { nidos de todas las } \\
\text { materias en cada } \\
\text { uno de los niveles, } \\
\text { indicando el módulo } \\
\text { y área a la que perte- } \\
\text { necen, así como el } \\
\text { número de horas y } \\
\text { créditos académicos }\end{array}$ & $\begin{array}{l}\text { La materia de } \\
\text { Liderazgo en sí misma } \\
\text { forma parte del } \\
\text { módulo de Liderazgo } \\
\text { Militar, el cual } \\
\text { forma parte del área } \\
\text { Profesional General, } \\
\text { con } 32 \text { horas y } 2 \\
\text { créditos académicos. }\end{array}$ & $\begin{array}{l}\text { Los temas de género } \\
\text { se abordan en las } \\
\text { materias Derecho } \\
\text { Constitucional y DD. } \\
\text { HH., y en Psicología I, } \\
\text { de primero y segundo } \\
\text { nivel, ambas del área } \\
\text { Socio Humanística }\end{array}$ \\
\hline
\end{tabular}




\begin{tabular}{|c|c|c|c|}
\hline $\begin{array}{l}\text { Identificación del } \\
\text { documento }\end{array}$ & Descripción general & Liderazgo militar & Género \\
\hline & & $\begin{array}{l}\text { Contenidos: La base } \\
\text { del liderazgo, Roles, } \\
\text { niveles y condiciones } \\
\text { especiales del liderazgo, } \\
\text { y Atributos del líder } \\
\text { del Ejército. } \\
\text { La materia de } \\
\text { Liderazgo ante la } \\
\text { Sociedad es parte del } \\
\text { mismo módulo y } \\
\text { área, con } 30 \text { horas y } \\
\text { un crédito. Presenta } \\
10 \text { contenidos sobre } \\
\text { confianza, ética, nego- } \\
\text { ciación y resolución de } \\
\text { conflictos, entre otros }\end{array}$ & \\
\hline $\begin{array}{l}\text { Syllabus de Liderazgo } \\
\text { en sí mismo }\end{array}$ & $\begin{array}{l}\text { Presenta información } \\
\text { general y especí- } \\
\text { fica del saber, las } \\
\text { competencias general } \\
\text { y específicas que } \\
\text { desarrolla. Los conte- } \\
\text { nidos, temas, estra- } \\
\text { tegias, indicadores, } \\
\text { evidencias y lecturas } \\
\text { obligatorias y comple- } \\
\text { mentarias }\end{array}$ & $\begin{array}{l}\text { Competencia general: } \\
\text { evidencia conoci- } \\
\text { miento claro de las } \\
\text { bases para formarse } \\
\text { como un líder militar } \\
\text { del futuro, a fin de } \\
\text { ejercer influencia } \\
\text { positiva en su entorno } \\
\text { con el propósito de } \\
\text { alcanzar el estado final } \\
\text { deseado }\end{array}$ & No registra \\
\hline $\begin{array}{l}\text { Syllabus de Liderazgo } \\
\text { ante la sociedad }\end{array}$ & $\begin{array}{l}\text { Presenta información } \\
\text { general y especí- } \\
\text { fica del saber, las } \\
\text { competencias general } \\
\text { y específicas que } \\
\text { desarrolla. Los conte- } \\
\text { nidos, temas, estra- } \\
\text { tegias, indicadores, } \\
\text { evidencias y lecturas } \\
\text { obligatorias y comple- } \\
\text { mentarias. }\end{array}$ & $\begin{array}{l}\text { Competencia general: } \\
\text { Muestra a través de sus } \\
\text { actos, los rasgos que lo } \\
\text { identifican como un } \\
\text { líder en la sociedad, } \\
\text { aceptando con una } \\
\text { clara convicción, el } \\
\text { sentido de la misión, } \\
\text { la historia y la nación, } \\
\text { como una forma de } \\
\text { vida orientada hacia el } \\
\text { servicio público } \\
\text { Se dicta en inglés }\end{array}$ & No registra \\
\hline
\end{tabular}

Fuente: Original de las autoras. 
En coherencia con los lineamientos de la política pública sectorial para avanzar hacia la perspectiva de género, se debe asumir de forma directa el reto de vincular los progresos alcanzados desde la Oficina de Género del Ejército Nacional con sus procesos de capacitación, formación y entrenamiento, en las discusiones académicas que "se alinean con la política de una Fuerza multimisión, al que apunta el Ejército Nacional en su proceso de transformación permanente" (Ministerio de Defensa, 2018, p. 42).

\section{Currículo integrador, la inclusión del género}

De acuerdo con la revisión documental, para alcanzar la integralidad — por lo menos desde los temas que se tratan en este trabajo - en el estudio de la formación militar es necesario incluir procesos de análisis, por lo cual se acude a la perspectiva crítica del currículo como un soporte conceptual y metodológico.

\section{Perspectiva Renovadora}

Abordar el currículo desde una perspectiva renovadora y crítica pone en el centro de los procesos a los sujetos y los actores que intervienen en la formación, lo cual implica

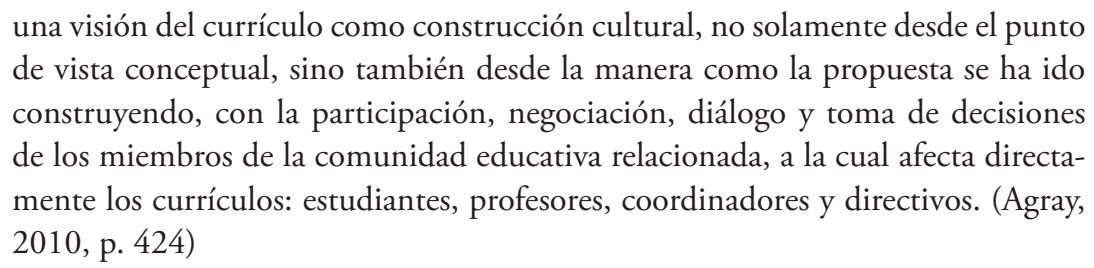

Desde esta perspectiva el currículo es una oportunidad para formar sujetos éticos y políticos que generen estrategias de análisis de su realidad desde estrategias integrales e incluyentes.

\section{Propuesta en Nueve pasos, un aVANCe hacia el diseño Curricular}

Existen diferentes formas de generar currículos desde la perspectiva crítica y renovadora. Para el proceso propiamente dicho de la ESMIC, y de acuerdo con sus dinámicas y posibilidades, la propuesta de los nueve pasos es una manera de materializar el avance, los retos y las perspectivas del diseño curricular en cuanto a liderazgo y género. Esta propuesta puede ser un insumo importante para que la institución la desarrolle en la práctica.

Los nueve pasos contemplan un recorrido procesual que inicia con la comprensión del estado actual de la propuesta curricular y finaliza con el ejercicio 
de orientación, como una opción de acompañar la implementación de los cambios establecidos a partir de la evaluación continua (Roa, 2019). Este proceso implica garantizar unas condiciones iniciales en cuanto a los actores que intervienen, la asignación de tiempos para la gestión del proceso, el acceso a documentos institucionales, la postura crítica, contextualizada y propositiva, frente a lo que se busca incluir en los currículos y el ejercicio de documentación de cada una de las etapas del proceso. Cada paso contempla objetivos, retos, metodología y producto.

- Paso 1. Comprender: esta etapa se dirige a recuperar la trayectoria del programa y el marco institucional en relación con la propuesta curricular actual. Se hace énfasis en dar cuenta de lo que se está implementando en comparación con lo que se diseñó.

- Paso 2. Ubicar: esta etapa se orienta a encontrar los aspectos microcurriculares a partir de la identificación de actores clave, documentos, experiencias de aula, en un intento por construir la memoria colectiva del programa. Está relacionada con la fase de análisis previo.

- Paso 3. Revisar: en este punto se da cuenta de cuáles son los aspectos para mejorar, mantener y cambiar en pro del ajuste curricular. Esta etapa da cierre al análisis previo y recoge las orientaciones para pasar al diseńo curricular propiamente dicho.

- Paso 4. Reconocer: en este paso se busca identificar categorías, recuperar buenas prácticas, experiencias y propuestas de lo que se ha revisado en el marco del trabajo colectivo, que den respuesta a la fase anterior y que permitan visibilizar la articulación de las trayectorias del programa y el ajuste que se va a realizar.

- Paso 5. Interpretar: en este se discute en torno al referente epistemológico y conceptual que sustentará la propuesta. Se busca así tejer la propuesta desde los referentes conceptuales, incluyendo el ámbito disciplinar y profesional.

- Paso 6. Construir: en esta fase se plasma la elaboración de la propuesta de ajuste propia, la cual hace referencia al trabajo colectivo, el análisis y comprensión de la trayectoria curricular del programa, además de especificar cada una de las demandas específicas que hace la normatividad vigente, en relación con el proceso de formación profesional.

- Paso 7. Unificar: esta etapa corresponde a la fase de aplicación curricular, en la cual se parte de socializar e integrar referentes institucionales, normatividad vigente e intereses del colectivo a la propuesta diseñada, en pro de iniciar la implementación. 
- Paso 8. Liderar: luego de socializado con la comunidad académica, corresponde implementar el proceso y gestionar las condiciones necesarias para que se lleve a cabo.

- Paso 9. Orientar: esta etapa corresponde a la fase de evaluación, sobre la que es necesario hacer dos aclaraciones: la primera es que no se trata de un paso final, sino que se asume como un proceso que está presente desde el análisis previo y que tiene mayor relevancia luego de que se ha implementado la propuesta. La segunda es que para tomar decisiones que orienten las modificaciones a futuro es necesario hacer una evaluación que garantice el acompañamiento del proceso y realizar una lectura crítica de lo proyectado-diseñado-ejecutado.

\section{Propuesta de lineamientos}

El esquema metodológico para incluir en el currículo el liderazgo militar y género desde una perspectiva renovadora y crítica se basa en el proceso práctico y riguroso que propone Roa (2019). Sin embargo, los lineamientos son los insumos que enmarcan las principales directrices que se pueden discutir y analizar en ese proceso. En tal sentido, y teniendo en cuenta las creencias y actitudes sobre la formación y el ejercicio del liderazgo militar en la ESMIC, así como los resultados del análisis de la revisión documental, se proponen los siguientes lineamientos para los niveles macro- $\mathrm{y}$ microcurricular.

\section{Macrocurrículo}

En este nivel se plantean las competencias para el liderazgo militar con perspectiva de género, para lo cual se toma como base el marco teórico de las competencias de igualdad de género (Mimbrero et al., 2017) y las de liderazgo militar que establece el Proyecto Educativo del Programa de Ciencias Militares (ESMIC, 2019). De acuerdo con estos referentes, las competencias se clasifican en los niveles sociocultural, relacional y personal: las del primer nivel corresponden a los conocimientos en liderazgo femenino, competencias del saber; las del segundo nivel son competencias del hacer y el convivir en relación con este modelo de liderazgo, y las del nivel personal son competencias del ser. Como se evidencia en el Proyecto Educativo del Programa de Ciencias Militares (ESMIC, 2019), el liderazgo militar se entiende como una competencia general del convivir, que se desagrega en competencias específicas del saber, del ser, del hacer y del convivir. 
Nivel sociocultural. Competencias de Conocimientos en materia de género y liderazgo femenino (saber)

- Conoce materias relacionadas con la aplicación del enfoque de género al liderazgo militar.

- Identifica y sabe utilizar herramientas para aplicar la transversalidad de género al liderazgo militar.

- Reconoce las situaciones que viven mujeres y hombres en el ejercicio del liderazgo militar.

- Valora positivamente las propuestas orientadas a combatir los desequilibrios existentes entre mujeres y hombres en materia de liderazgo.

Nivel relacional. Competencias metodológicas (hacer)

- Contextualiza el uso de conocimientos adquiridos a situaciones reales en las que interactúan hombres y mujeres en el ejercicio del liderazgo militar.

- Propone medidas orientadas a incorporar elementos de liderazgo femenino aplicables al contexto actual de la formación militar.

- Plantea soluciones para superar desequilibrios de género existentes en la formación y ejercicio del liderazgo militar.

- Fomenta la participación y el desarrollo de sus subordinados en búsqueda de la excelencia.

- Escucha y analiza las ideas de sus subalternos, aceptando sus contribuciones siempre que sea posible y práctico hacerlo.

Competencias de participación (convivir)

- Supera resistencias emocionales que subjetivamente aparecen cuando se accede al conocimiento sobre liderazgo desde una perspectiva de género.

- Asume modelos genéricos construidos en contextos determinados (organización laboral, sistema familiar...) para comprender, adaptarse, enfrentar o resolver situaciones de desigualdad entre hombres y mujeres en el campo del liderazgo militar.

- Busca soluciones conjuntas con objeto de resolver los desequilibrios de género en materia de liderazgo militar.

- Mediante el ejemplo, obtiene el respeto de sus subordinados para ejercer influencia.

- Promueve la comunicación con sus subordinados, estimulando y respaldando el uso de sus habilidades. 
- Muestra apoyo a sus subordinados de manera individual y promueve el desarrollo de la creatividad, siendo capaz de emocionar, despertar e inspirar a realizar un esfuerzo adicional para alcanzar los objetivos propuestos.

Nivel personal. Competencias personales (ser)

- Reconoce un cambio actitudinal y profesional propio vinculado a la integración del enfoque de género en la formación y el ejercicio del liderazgo militar.

- Observa cambios en el reconocimiento de la propia imagen personal alejado de esencialismos genéricos.

\section{Microcurrículo}

El microcurrículo se materializa en la interacción docente-estudiante-contexto-medioambiente e integra las actividades de aula propuestas en los planes de estudio de cada línea de formación (Roa, 2019). Con base en el trabajo de Arraiz et al. (2015), en este nivel se propone aplicar las siguientes pautas para los procesos de enseñanza-aprendizaje de liderazgo militar en el aula.

\section{Revisar el currículo}

$\mathrm{Al}$ respecto es importante entender que coexiste el currículo visible u oficial, que se transmite explícitamente en los cursos de un programa, y el currículo oculto, en el cual se transfieren de manera implícita conocimientos, valores y actitudes a lo largo de todo el proceso educativo, ambos con un impacto importante en la socialización de género. Por lo tanto, la revisión debe ser de ambos tipos de currículo para evidenciar la jerarquización de materias y valores asociados, considerados socialmente masculinos sobre aquellos que se asumen como femeninos. Sobre el currículo oculto también se debe tener en cuenta "el uso del lenguaje, la concepción y el uso de los espacios, el acceso y la utilización de los recursos, las relaciones con las compañeras y con los compañeros, las relaciones con el profesorado y entre el profesorado, la metodología de enseńanza-aprendizaje, etc." (Arraiz et al., 2015, p. 25). Asimismo, se debe tener claro que todos estos aspectos transmiten las expectativas en torno al lugar, rol, función, comportamiento, etc., de los futuros y futuras oficiales.

\section{Realizar un uso inclusivo del lenguaje}

Es fundamental adoptar medidas para hacer un uso incluyente y no sexista del lenguaje oral y escrito, ya que es el principal medio en el proceso de enseñanza-aprendizaje por el cual se transmiten conocimientos, valores y actitudes, además 
de que no solo refleja sino además construye la realidad. Esto suele implicar hacer una elaboración más precisa de los mensajes para reflejar mejor la realidad y construir una más justa y equitativa, sobre todo en un contexto en el que hay un predominio numérico de un sexo sobre el otro, como es el caso de la ESMIC.

\section{Diseñar y/o elegir materiales y libros de texto inclusivos y coeducativos}

Los contenidos de estos elementos, en cuanto a las imágenes y el lenguaje que emplean, constituyen representaciones de cierta realidad social, valores, formas de expresión, normas, etc., basadas en estereotipos de género que no representan la realidad actual en su totalidad. Es por ello que los textos deben brindar modelos de identificación que no discriminen por sexo, sino que, por el contrario, incorporen las características requeridas de ambos géneros para la formación de un liderazgo militar exitoso.

\section{Integrar la contribución social e histórica de las mujeres}

El tradicional estilo androcéntrico de enseñanza y aprendizaje deja a hombres y mujeres en formación sin referentes femeninos, lo que es muy evidente en el contexto del liderazgo militar, en cuya historia los hombres se ven como protagonistas, mientras que las mujeres aparecen en un papel secundario. Por esta razón es necesario visibilizar el papel de las mujeres en el liderazgo militar, de forma que sus aportes, habilidades, experiencias, etc., tengan el mismo reconocimiento que han tenido los de los hombres para el desempeño profesional.

\section{Realizar una orientación académico profesional no sexista}

De acuerdo con el punto anterior, la incorporación de elementos de liderazgo femenino al currículo comienza con reconocer su importancia para que hombres y mujeres ejerzan el liderazgo militar de manera eficiente, de la misma forma que tradicionalmente lo han tenido varios componentes del estilo masculino. $Y$ en esta misma vía, garantizar condiciones equitativas para ejercer el liderazgo militar en los diferentes escenarios de desempeño que ofrece y requiere la institución.

\section{Conclusión}

Si bien, como lo expresa Carreiras (2018), la Resolución 1325, aprobada por el Consejo de Seguridad de la Organización de las Naciones Unidas (ONU) en 2000, constituyó un hito en el reconocimiento internacional de la dimensión de género en los conflictos armados y el papel de las mujeres en su prevención y reso- 
lución, en el caso del Ejército Nacional de Colombia se considera que la evolución institucional ha sido la principal razón para incluir a las mujeres. No obstante, se han experimentado contradicciones y limitaciones en este proceso de incorporación debido a las connotaciones que tienen los conceptos de igualdad y diferencia cuando se habla de la participación de las mujeres en las Fuerzas Armadas.

Cabe señalar que se están desarrollando procesos de cambio en la institución militar que tienen que ver con la generación de espacios como el Observatorio de Género de la ESMIC, el cual mantiene una línea importante de investigación sobre las mujeres en el campo militar. Asimismo, desde las investigaciones se avanza en crear espacios de discusión sobre el papel de las mujeres y que a futuro pueden ser un elemento determinante en los procesos curriculares de formación. Sin embargo, se percibe resistencia para abordar el concepto de género y no ha habido la apertura institucional necesaria para realizar los procesos de transformación, por lo cual se debe hacer un llamado para incorporar el concepto en los escenarios militares.

Desde las instituciones de educación superior es necesario reflexionar acerca de las prácticas curriculares y proponer lineamientos que conduzcan a comprender los contextos, identificar y formular problemas, así como a diseñar estrategias para su solución. El análisis realizado en este trabajo permitió identificar que la investigación curricular debe estar presente en los procesos de cambio del Ejército Nacional, pues es necesario conceptualizar y proponer nuevas formas de aprendizaje desde escenarios de construcción colectiva con los diferentes actores que intervienen en la formación, lo cual permitirá orientar las decisiones que desde el currículo se deriven en las funciones de la ESMIC como institución de educación superior. En este sentido, la propuesta presentada en este estudio responde a las directrices emitidas por el Gobierno en cuanto a

emitir lineamientos para los procesos de formación y estudios en temas de género, así como revisar la malla curricular de las diferentes escuelas y proponer la inclusión de los temas relacionados con esta política, fortaleciendo el enfoque de derechos humanos, equidad de género, enfoque diferencial y derechos de las mujeres en los diferentes procesos de formación a integrantes de las Fuerzas Militares. (Ministerio de Defensa, 2018, p. 66)

En las condiciones actuales, en las que existen diferencias de género en la carrera militar como profesión, se espera que estos lineamientos puedan contribuir a que se cumpla la misión del Programa de "formar integralmente a los futuros oficiales del Ejército como líderes comandantes de pelotón, con sólidas competencias profesionales, investigativas y socio humanísticas", así como a "generar un 
modelo de liderazgo militar exitoso, que contribuya al comando y dirección de unidades militares, con vocación de servicio, principios, valores, capacidad emprendedora y comprensión del entorno nacional e internacional", tal como se plantea en sus objetivos específicos (ESMIC, 2019).

\section{Financiación}

Esta investigación ha sido realizada gracias al financiamiento de la ESMIC y Minciencias, a través de la Convocatoria No. 784 "Programa de estancias postdoctorales beneficiarios Colciencias 2017”. Las autoras declaran que no existe ningún potencial conflicto de interés relacionado con el capítulo.

\section{Referencias}

Agray, N. (2010). La construcción de currículo desde perspectivas críticas: una producción cultural. Signo y Pensamiento, 29 (56), 420-427.

Aktouf, O. (2001). La administración: entre tradición y renovación. Cali: Artes Gráficas del Valle.

Arraiz Argoitia, B. et al. (2015). Guia para la incorporación de la perspectiva de género en el currículum y en la actividad docente de las enseñanzas de régimen especial y de formación profesional. VitoriaGasteiz: Servicio Central de Publicaciones del Gobierno Vasco.

Avendańo, W. \& Parada, A. (2013). El currículo en la sociedad del conocimiento. Educación y Educadores, 16 (1), 159-174.

Bass, B. M. (1985). Leadership and performance beyond expectations. Nueva York: Free Press.

Boldry, J., Wood, W. \& Kashy, D. (2001). Gender stereotypes and the evaluation of men and women in military training. Journal of Social Issues, 57 (4), 689-705. doi:10.1111/0022-4537.00236

Camacho, C. (2014). Ejército, feminidades y géneros performativos. Las experiencias de ser mujer y militar en la Escuela Militar de Cadetes "General José María Córdova” (Tesis de Maestría en Estudios Culturales). Pontificia Universidad Javeriana, Bogotá, D. C., Colombia.

Camacho, C., Pérez, L., Rincón, L., Muñoz, A. \& Ramírez, C. (2016). Evaluación curricular. Realidades y desafios. Bogotá: Universidad de La Salle.

Carreiras, H. (2018). La integración de género en las Fuerzas Armadas. Condicionamientos y perspectivas. Nueva Sociedad, (278), 130-143.

Castillejo, A. (2015). Utopía y desarraigo. Papeles del CEIC. International Journal on Collective Identity Research, 1 (121), 1-30. http://dx.doi.org/10.1387/pceic.13036

Centro de Doctrina del Ejército [Cedoe]. (2016). Manual Fundamental del Ejército 6-22. Liderazgo. Ejército Nacional de Colombia.

Centro de Doctrina del Ejército [Cedoe]. (2017). Manual Fundamental de Referencia del Ejército 6-22. Liderazgo. Ejército Nacional de Colombia.

ComandoGeneraldelasFuerzasMilitares.(2015). DirectivaPermanente20151110000277-MDN-CGFMJEMC-SEMCFJI-JEDHDIH-23.1. Lineamientos estratégicos para las Fuerzas Militares en Equidad 
de Género, Enfoque Diferencial y Prevención de Violencias Basadas en Género. Bogotá: Comando General Fuerzas Militares.

Cuadrado, I. (2003). ¿Emplean hombres y mujeres diferentes estilos de liderazgo? Análisis de la influencia de los estilos de liderazgo en el acceso a los puestos de dirección. Revista de Psicología Social, 18 (3), 283-307.

Echavarría, C. (1994). Apuntes sobre la integración de la mujer en las Fuerzas Armadas. Cuadernos de Estrategia, (68), 67-86.

Escuela Militar de Cadetes “General José María Córdova” [ESMIC]. (2019). Proyecto Educativo del Programa de Ciencias Militares.

Fernández-Osorio, A., Latorre, E. \& Mayorga, N. (2018). The 2018 Colombian Military Academy dataset: a sociological study of population. Revista Cientifica General José Maria Córdova, 16 (23), 147-162. http://dx.doi.org/10.21830/19006586.345

Formujer (2003). Género y formación por competencias: aportes conceptuales, herramientas y aplicaciones. Montevideo: Cinterfor y Organización Internacional del Trabajo.

García, V. (2014). Estereotipos de género y liderazgo femenino. Ponencia presentada en el VI Congreso Internacional de Investigación y Práctica Profesional en Psicología, XXI Jornadas de Investigación, Décimo Encuentro de Investigadores en Psicología del Mercosur. Facultad de Psicología, Universidad de Buenos Aires, Argentina.

Gimeno Sacristán, J. (1999). La construcción del discurso acerca de la diversidad y sus prácticas. Aula de Innovación Educativa, 81, 69-72.

Gimeno Sacristán, J. (2010). Saberes e incertidumbres sobre el currículum. Madrid: Morata.

Kaufmann, A. (1996). Tercer milenio y liderazgo femenino. En L. Nuńo (coord.), Mujeres: de lo privado a lo público (pp. 177-186). Madrid: Tecnos.

López, I. (2007). El enfoque de género en la intervención social. Madrid: Cruz Roja.

Lupano, M., Castro Solano, A. \& Casullo, M. (2008). Prototipos de liderazgo masculino y femenino en población militar. Revista de Psicología, 26 (2), 195-218. Recuperado de http://revistas.pucp. edu.pe/index.php/psicologia/article/view/1057/1021

Malagón, L., Machado, D. \& Rodríguez, H. (2013). Currículo y politicas públicas educativas. Ibagué: Universidad del Tolima.

Medina-Vicent, M. (2015). Aproximación al estudio del liderazgo femenino a través del modelo transformacional. En Investigació i gènere a la Universitat Jaume I (Colecció Humanitats 46 (pp. 187-192). Recuperado de http://repositori.uji.es/xmlui/bitstream/handle/10234/158386/ Aproximacion_al_estudio_del_Liderazgo_Fe.pdf?sequence=1

Mimbrero, C., Pallarès, S. \& Cantera L. (2017). Competencias de igualdad de género: capacitación para la equidad entre mujeres y hombres en las organizaciones. Athenea Digital, 17(2), 265-286. http://dx.doi.org/10.5565/rev/athenea.1609

Ministerio de Defensa Nacional. (2010). Sistema Educativo de las Fuerzas Armadas (SEFA). Lineamientos Curriculares Fuerzas Armadas. Bogotá, D. C.: Imprenta y Publicaciones FF. MM.

Ministerio de Defensa Nacional. (2018). Política Pública Sectorial de Transversalización del Enfoque de Género para el Personal Uniformado de la Fuerza Pública (2018-2027) [documento en línea]. Recuperado de https:/www.justiciamilitar.gov.co/irj/go/km/docs/Mindefensa/Documentos/ descargas/Prensa/Documentos/politica_genero.pdf 
Ministerio de Educación Nacional. (2016). Glosario. Lineamientos curriculares. Recuperado de https:// www.mineducacion.gov.co/1621/article-80860.html

Miranda, M. (2012). Diferencia entre la perspectiva de género y la ideología de género. Díkaion, 21 (2), 337-356.

Osorio, C (2012). El microcurrículo como contexto de complejidad en la educación posgradual. Ponencia presentada en el V Congreso Internacional de Transdisciplinariedad, Complejidad y Ecoformación "Emergencia de una educación integral de calidad para la transformación social". Universidad Simón Bolívar, Barranquilla, Colombia.

Ospina, J (2006). La motivación, motor del aprendizaje. Revista Ciencias de la Salud, (4), 158-160.

Parada, G. (2015). Liderazgo transformacional: un reto en la cultura organizacional del Ejército Colombiano (Trabajo de grado). Universidad Militar Nueva Granada. Bogotá, D. C., Colombia.

Perilla-Toro, L.E. \& Cabrera, L. J. (2018). Caracterización del liderazgo militar desde una perspectiva de género. En A. Fernández-Osorio \& J. Latorre (eds.), La construcción del rol de la mujer militar (pp. 67-91). Bogotá, D. C.: Sello Editorial ESMIC.

Ramos, A. (2005). Mujeres y liderazgo: una nueva forma de dirigir. Valencia: PUV.

República de Colombia. (1994). Ley 115 de 1994. Congreso de la República. Diario Oficial 41.214.

Roa Mendoza, C. P. (2019). Propuesta de lineamientos curriculares en educación superior desde la perspectiva del currículo crítico. Una alternativa a partir de los desafíos de la formación profesional en Trabajo Social (Tesis doctoral). Universidad de La Salle, Bogotá, D. C., Colombia.

Rojas, A. M. C. \& Palacio, I. C. C. (2004). Análisis del concepto enseñanza en las teorías curriculares de Lawrence Stenhouse y José Gimeno Sacristán. Educación y Pedagogía, 16 (40), 141-152.

Sampieri, R. H. \& Mendoza, Ch. (2018). Metodología de la investigación: las rutas cuantitativa, cualitativa y mixta. México: McGraw Hill.

Tovar, M. C. \& Sarmiento, P. (2011). El diseño curricular, una responsabilidad compartida. Colombia Médica, 42 (4), 508-517. 